FOUNDATIONS OF COMPUTING AND DECISION SCIENCES

Vol. 41

(2016)

No. 4

DE DE GRUYTER OPEN

\title{
HANDLING THE MULTIPLICITY OF SOLUTIONS IN A MOEA BASED PDA-THESEUS FRAMEWORK FOR MULTI-CRITERIA SORTING
}

\author{
Edgar COVANTES*, Eduardo FERNÁNDEZ**, Jorge NAVARRO***
}

\begin{abstract}
This paper proposes the combination of the THESEUS multi-criteria sorting method with an evolutionary optimization-based preference-disaggregation analysis. The main features of the combined method are studied by performing an extensive computer experiment that explores many models of preferences and sizes of problems as well as different degrees of decision-maker involvement. As a result of the experiment, the effectiveness of the combined framework and the importance of the decision-maker's involvement are characterized.
\end{abstract}

Keywords: multi-criteria decision; fuzzy outranking relations; parameter elicitation; evolutionary algorithms.

\section{Introduction}

Multi-criteria sorting (or multi-criteria ordinal classification) is a particular case of classification problems that has received increasing interest in recent years. Unlike nominal classification, sorting refers to problems in which the categories have been defined in an ordinal way.

Multi-criteria methods entail a decision-maker $(D M)$ reflecting his/her preferences in a pre-specified mathematical structure. Hence, obtaining preference information from the $D M$ and formalizing this information by using preferential parameters is a crucial aspect in building a multi-criteria decision model ([5]). The development of these models can be based on direct or indirect elicitation procedures. In the first case, the DM must specify

* Autonomous University of Sinaloa, Faculty of Civil Engineering, Blvd. Las Americas, Ciudad Universitaria Culiacan, Sinaloa, Mexico CP. 80040.

** Autonomous University of Sinaloa, Faculty of Civil Engineering, Blvd. Las Americas, Ciudad Universitaria Culiacan, Sinaloa, Mexico CP. 80040, e-mail: eddyf@uas.edu.mx, Tel/Fax: (52)667 7134053.

*** Autonomous University of Sinaloa, Faculty of Computer Science, Josefa Ortiz de Dominguez Ote., Ciudad Universitaria, Culiacan, Sinaloa, Mexico CP. 80040. 
preferential parameters through an interactive process guided by a decision analyst in which the cognitive difficulties are an important concern. On the other hand, indirect procedures, which use the so-called preference-disaggregation analysis $(P D A)$, use regression-like methods for inferring the set of parameters from a battery of decision examples ([6]).

In the framework of Multiple Criteria Decision Aid (MCDA), Jacquet-Lagreze and Siskos pioneered the UTA method in the early 1980's ([14]). Indirect elicitation methods still capture the interest of the research field community (e.g. [23]). According to [13], MCDA approaches based on disaggregation paradigms are of increasing interest because they require relatively less cognitive effort from the $D M$. The direct eliciting method has been criticized by (among others) Marchant (Communication in 71st Meeting of the Euro Working Group on Multiple Criteria Decision Aiding, Turin 2010) and Pirlot (Communication in 71st Meeting of the Euro Working Group on Multiple Criteria Decision Aiding, Turin 2010). Marchant stated that the only valid preferential input-information is that arising from the DM's preferential judgments about actions or pairs of actions. These criticisms are even more important in the frame of outranking methods, because the DM must set parameters that are very unfamiliar to him/her (e.g. veto thresholds).

Although several authors (e.g. [6]) emphasize the importance of a co-constructive process between the $D M$ and the decision-analyst to obtain a final parameter setting, to the best of our knowledge there is no published study that addresses this issue in a systematic and quantitative fashion.

The multiplicity of solutions is an important concern for PDA methods. These use an error optimization model for fitting the preference parameters that reproduce the reference set. When the reference information is relatively scarce, many sets of preference parameters may correspond to the same minimal error ([24]). This makes it necessary to study the severity of the problem of the multiplicity of preference parameters, and how this problem is influenced by the characteristics of the data.

Here, we are interested in combining a disaggregation approach based on evolutionary multi-objective optimization with the THESEUS multi-criteria sorting method proposed in [9]. THESEUS uses a set $T$ of training examples (reference actions) that contains implicit information about the assignment policy from the $D M$. His/her preferences are represented by an index of credibility of the outranking $\sigma$ that models the degree of credibility of the outranking. As in [15] and [19], given a certain credibility threshold $\lambda$, the general approach of THESEUS is based on the fulfilment of the implications

$$
\begin{aligned}
& \forall \boldsymbol{b} \in T \\
& \sigma(x, \boldsymbol{b}) \geq \lambda \Rightarrow C(x) \gtrsim C(\boldsymbol{b}) \\
& \sigma(\boldsymbol{b}, x) \geq \lambda \Rightarrow C(\boldsymbol{b}) \gtrsim C(x)
\end{aligned}
$$

(where $C(\boldsymbol{b})$ denotes the category to which the object $\boldsymbol{b}$ was assigned; $C(x)$ is the most appropriate assignment of $x$; and $\succsim$ denotes the statement "at least as good as" on the set of categories, which is related to the decision-aiding context).

$\sigma$ may be built as in ELECTRE III, ELECTRE TRI, PROMETHEE and other outranking methods (e.g. [20]); its parameters may be set directly or indirectly. However, THESEUS requires one to work with a pair $(\sigma, \lambda)$ that is as consistent as possible with Eqs. (1). This claim for consistency requires the use of indirect elicitation methods in the first step of THESEUS, prior to the assignment decisions.

In the present paper, THESEUS is extended in order to become a multi-criteria PDAsorting method. We are interested in handling the multiplicity of parameter settings that are 
compatible with the preference information provided by the $D M$. We use an evolutionary multi-objective approach to find a set of Pareto optimal points that are potential solutions to the inference problem. The analyst- $D M$ couple could test the alternative settings by using assignments not belonging to the original reference set, but this process could be very demanding. As alternative, we propose to consider a particular Pareto optimal setting that can be detected automatically; no effort from the $D M$ is needed in order to identify this point. Then, we perform an extensive experiment in which the classification accuracy associated with that point is compared with the closest points to the parameter values assumed in the simulation study. Some answers are obtained to the following questions:

1. To what extent is the quality of solutions affected by the number of categories and criteria?

2. To what extent is the quality influenced by the cardinality of the reference set?

3. To what extent is the quality influenced by the DM's agreement to express his/her preferences and beliefs, participating in a co-constructive process with the decisionanalyst?

4. How far can the quality of the final solution be improved by a comparison process between different potential solutions?

The first two questions have recently been addressed in [7] and [24] in the frame of value functions, and in [6] for ELECTRE TRI. To the best of our knowledge, there is no published paper that expressly approaches the last two questions concerning PDA in outranking methods.

The paper is structured as follows. The background is presented in Section 2. This section includes several premises, some notation and some definitions; the THESEUS method and the evolutionary approaches to $P D A$ are briefly outlined in this section. Our proposal is introduced in Section 3. Section 4 presents the experiment in which the proposal is explored for a very wide range of simulated decision-maker preferences, categories, criteria, and reference set cardinalities. Some conclusions are given in Section 5.

\section{Background}

\subsection{Basic premises and definitions}

Following [11] and [12], let us propose some premises and definitions:

i) There is a finite set of ordered categories $\boldsymbol{C} \boldsymbol{t}=\left\{C_{1}, \ldots C_{\mathrm{M}}\right\},(M \geq 2) ; C_{M}$ is assumed to be the preferred category. The term 'preferred' is related to each particular sorting problem (for instance, 'higher quality', 'more consensual', 'less risky')

ii) Let $U$ be the universe of objects (actions) $x$ described by a coherent set of $N$ criteria, denoted $G=\left\{g_{1}, g_{2}, \ldots, g_{j}, \ldots, g_{N}\right\}$, with $N \geq 3$.

iii) There is a $D M$ who has (or agrees with) a certain decision policy defined on a subset $U$ ' of the universe.

iv) There is a set of reference actions or training examples $T$, which is composed of elements $b_{k j} \in U$, assigned to category $C_{k},(k=1, \ldots M)$ (the second subindex $(j)$ denotes the $j$-th object in the corresponding category denoted by the first subindex). $T$ is created or approved by the $D M$. 
v) There is an index of credibility of outranking $\sigma(x, y)$ defined on $U \times U$. Its value can be interpreted as the degree of credibility of the statement ' $x$ is at least as good as $y$ ' from the DM's perspective in each particular sorting problem (for instance, ' $x$ is at least as consensual as $y$ ', or ' $x$ is at most as risky as $y$ ').

vi) The $D M$ agrees with certain indifference, strict preference, weak preference and outranking relations on subsets of $U$ denoted by $I, P, Q$, and $S$, respectively. These were defined in [21] as follows:

- Indifference corresponds to the existence of clear and positive reasons that justify equivalence between the two actions. Notation: $x I y$.

- Strict preference corresponds to the existence of clear and positive reasons that justify a significant preference in favour of one (identified) of the two actions. The statement ' $x$ is strictly preferred to $y$ ' is denoted by $x P y$.

- Weak preference corresponds to the existence of clear and positive reasons in favour of $x$ over $y$, but these reasons are not sufficient to justify strict preference. Indifference and strict preference cannot be distinguished appropriately. This is denoted by $x Q y$.

- Outranking: It corresponds to the existence of clear and positive reasons that justify the statement ' $x$ is at least as good as $y$ ', but with no significant division being established among the situations of strict preference, weak preference and indifference. Notation: $x S y$.

\section{Definition 1:}

The assignment of $x \in U-T$ to category $C(x)$ is said to be consistent with $T$ and the DM's preference relations if

$$
\forall b_{k j} \in T
$$

$x$ outranks $b_{k j} \Rightarrow C(x)$ is at least as good as $C_{k}$

$b_{k j}$ outranks $x \Rightarrow C_{k}$ is at least as good as $C(x)$

A consistent $D M$ should make consistent assignments in the sense of Definition 1 . Nevertheless, inconsistent assignments may be a consequence of inadequate assignments of some reference objects and the non-transitivity of the DM's outranking relation.

Let us consider a real value $\lambda>0.5$.

\section{Definition 2:}

Given $\sigma(x, y)$, the following crisp binary relations are defined on the universe:

$(x, y) \in S(\lambda)$ iff $\sigma(x, y) \geq \lambda$ ( $\lambda$-outranking)

$(x, y) \in P(\lambda)$ iff $\sigma(x, y) \geq \lambda \wedge \sigma(y, x)<0.5$ ( $\lambda$-strict preference)

$(x, y) \in Q(\lambda)$ iff $\sigma(x, y) \geq \lambda \wedge 0.5 \leq \sigma(y, x)<\lambda$ ( $\lambda$-weak preference)

$(x, y) \in I(\lambda)$ iff $\sigma(x, y) \geq \lambda \wedge \sigma(y, x) \geq \lambda$ ( $\lambda$-indifference)

$(x, y) \in R(\lambda)$ iff $\sigma(x, y)<\lambda \wedge \sigma(y, x)<\lambda$ ( $\lambda$-incomparability)

\section{Definition 3:}

The assignment of $x$ is said to be $(T, \sigma, \lambda)$ - consistent if

$\forall b_{k j} \in T$

$x S(\lambda) b_{k j} \Rightarrow C(x)$ is at least as good as $C_{k}$

$b_{k j} S(\lambda) x \Rightarrow C_{k}$ is at least as good as $C(x)$ 


\section{Definition 4:}

A reference set $T$ will be called $(\sigma, \lambda)$-consistent if

$\forall\left(b_{k j}, b_{n i}\right) \in T \times T$

$b_{k j} P(\lambda) b_{n i}$ or $b_{k j} Q(\lambda) b_{n i} \Rightarrow k \geq n$

$b_{k j} I(\lambda) b_{n i} \Rightarrow k=n$

\subsection{Some essential aspects of the THESEUS method}

Let us recall from [9], [11] and [12] a brief description of the THESEUS method. THESEUS is based on comparing a new object to be assigned with the objects in $T$ through $P(\lambda), Q(\lambda), I(\lambda)$. Suppose that $C(x)$ denotes a potential assignment of the object $x$. According to THESEUS, $C(x)$ should satisfy:

$$
\begin{aligned}
& \forall b_{k j} \in T \\
& x P(\lambda) b_{k j} \Rightarrow C(x) \succsim C_{k} \\
& b_{k j} P(\lambda) x \Rightarrow C_{k} \succsim C(x) \\
& x Q(\lambda) b_{k j} \Rightarrow C(x) \succsim C_{k} \\
& b_{k j} Q(\lambda) x \Rightarrow C_{k} \succsim C(x) \\
& x I(\lambda) b_{k j} \Rightarrow C(x)=C_{k}
\end{aligned}
$$

(where $\gtrsim$ denotes the statement 'is at least as good as' on the set of categories).

Equations (4.a-c) express the required consistency amongst the preference model $\sigma$, the reference set and the appropriate assignments of $x$. If $S(\lambda) \subseteq S$, any assignment that is inconsistent with Eqs. (4.a-c) would produce dissatisfaction to a consistent $D M$.

The assignment $C(x)$ should be as compatible as possible with the current knowledge about the assignment policy, that is contained in $\sigma$ and $T$. The inconsistencies with Equations (4.a-c) are used by THESEUS to compare the potential assignments of $x$. Specifically:

- The set of $P(\lambda)$-inconsistencies for $x$ and $C(x)$ is defined as $A_{P}=\left\{\left(x, b_{k j}\right),\left(b_{k j}, x\right), b_{k j} \in T\right.$ such that (4.a) is FALSE\};

- The set of $Q(\lambda)$-inconsistencies for $x$ and $C(x)$ is defined as $A_{Q}=\left\{\left(x, b_{k j}\right)\right.$, $\left(b_{k j}, x\right), b_{k j} \in T$ such that (4.b) is FALSE $\}$;

- The set of $I(\lambda)$-inconsistencies for $x$ and $C(x)$ is defined as $A_{I}=\left\{\left(x, b_{k j}\right),\left(b_{k j}, x\right), b_{k j} \in T\right.$ such that (4.c) is FALSE .

The above inconsistencies are grouped in different levels of importance. In order to clarify this issue, let us suppose that $C(x)=C_{k}$ and consider $b_{m j} \in T$. Cases in which $x I(\lambda) b_{m j} \wedge|k-m|=1$ can be consequences of a certain 'discontinuity' of the description; $x$ may be close to the upper (lower) boundary of $C_{k}$ and $b_{m j}$ may be close to the lower (upper) boundary of $C_{j}$. These are called second-order $I(\lambda)$-inconsistencies and grouped in the set $A_{2 I}$. The set $A_{1 I}=A_{I}-A_{2 I}$ contains the so-called first-order $I(\lambda)$-inconsistencies, which are not consequences of the discontinuity effect described. Let $n_{P}, n_{Q}, n_{1 I}, n_{2 I}$ be the cardinality of the above-defined inconsistency sets, and $N_{1}=n_{P}+n_{Q}+n_{1 I}, N_{2}=n_{2 I}$. 
The THESEUS' assignment minimizes the above inconsistencies with lexicographic priority favouring $N_{1}$, which is considered the most important criterion. The THESEUS assignment rule follows:

For each $x \in U$ and given $\lambda>0.5$

a) Starting with $k=1(k=1, \ldots . M)$ and considering each $b_{k j} \in T$, calculate $N_{1}\left(C_{k}\right)$;

b) Identify the set $\left\{C_{j}\right\}$ whose elements hold $C_{j}=\operatorname{argmin} N_{1}\left(C_{k}\right)$;

c) Select $C_{k^{*}}=\operatorname{argmin} N_{2}\left(C_{i}\right)$;

$\left\{C_{j}\right\}$

d) If $C_{k^{*}}$ is a single solution, assign $x$ to $C_{k^{*}}$; the other case is approached below.

The suggestion may be a single category or a set of categories. The first case is called a well-defined assignment; otherwise, the solution obtained highlights the highest category $\left(C_{H}\right)$ and the lowest category $\left(C_{L}\right)$ that are appropriate for assigning the object, but fails in determining the most appropriate ([9]). Such solution is called 'a vague assignment'. In order to characterize well-defined assignments, the following Uniqueness Theorem is important:

Theorem: A THESEUS solution $C_{k^{*}}$ is unique only if one of the following conditions holds:

i) there is $(l, m)$ such that $x S(\lambda) b_{k^{* l}}$ and also $b_{k^{*} m} S(\lambda) x$;

ii) there is a $b_{M L}$ such that $x S(\lambda) b_{M L}$;

iii) there is a $b_{1 j}$ such that $b_{1 j} S(\lambda) x$.

In case ii) $C_{k^{*}}=C_{M}$; in iii) $C_{k^{*}}=C_{1}$.

(See the proof in [9]).

In a simple interpretation of the above theorem, in order to be unique the THESEUS assignment should be 'bounded' by two objects in the same category, or be 'bounded' by objects belonging to extreme categories. Thus, if no condition of the Uniqueness Theorem is satisfied by $x$, then its assignment is vague or ill-defined. As stated in [12], a pair $\left(b_{k l}\right.$, $b_{k m}$ ) with $x S(\lambda) b_{k l}$ and $b_{k m} S(\lambda) x$ is an information element that supports the assignment of $x$ to $C_{k}$. In the same way, reference actions fulfilling Conditions ii) or iii) are also information elements that support the assignment of $x$ to $C_{M}$ or $C_{1}$.

\subsection{Evolutionary-based $P D A$ approaches}

According to Premise v) in Section 2.1, our interest here is restricted to PDA in ELECTRE methods, which are among the most popular multi-criteria decision tools. In ELECTREbased models, inferring all the parameters simultaneously requires one to solve a non-linear programming problem with non-convex constraints, which is usually difficult (cf. [17]). According to [6], the relational form of these models and the veto conditions may make it impossible to infer the model parameters in real-size data sets. Otherwise, in small data sets the non-linear problem may be ill-determined; there are many different parameter settings that are compatible with the preference information, but no mathematical programming technique is able to describe the whole set of compatible parameter settings. The problem is particularly difficult when considering the effects of reinforced preference on the credibility of outranking as in [22].

In recent years, evolutionary algorithms have rendered powerful tools for solving difficult problems in a variety of fields and, in particular, for the treatment of non-linearity 
and global optimization in polynomial time ([2]). Three papers have examined the problem of inferring outranking model parameters by evolutionary techniques. In [6], Doumpos et al. used a differential evolution algorithm for inferring parameter values in the ELECTRE TRI method. In [8], Fernandez et al. proposed an evolutionary multi-objective algorithm for inferring the parameters of a fuzzy indifference relation model for multi-criteria sorting purposes. This method was extended in [10] in order to handle scarce preference information and the effect of reinforced preference in a more general decision context. The multi-objective approach, (though more complex), is more flexible than single-objective optimization because it allows a richer modelling of preferences. The solution to the parameter inference problem must satisfy several constraints in the parameter space. The $D M$ may be unable to make a direct elicitation of the model parameters, but (s)he may provide subjective information about criterion importance and parameter value ranges. As constraints, these judgments can reduce the search space and help to produce more acceptable solutions. As an additional advantage, an evolutionary multi-objective algorithm is capable of generating many good compromise solutions in the associated parameter space. As a result of the evolutionary exploration process, a characterization of the complete set of different model parameter settings is achieved. This information may be used to obtain a final parameter setting.

In [8] and [10], the objective functions measure the number of inconsistencies with preference relations in a similar way to Eqs. (4) and Section 2.2. Hence, in order to create a unified PDA-THESEUS framework for multi-criteria sorting, this approach seems to be desirable.

\section{A combined PDA-THESEUS framework}

\subsection{Finding potential parameter settings via multi-objective evolutionary optimization}

Let us denote by $\eta$ the set of the $\sigma$ - model's parameters and by $\lambda$ the credibility threshold. Consider the following logical implications:

$$
\begin{aligned}
& \forall\left(b_{k h}, b_{j i}\right) \in T \times T \\
& b_{k h} P(\eta, \lambda) b_{j i} \Rightarrow k \geq j \\
& b_{k h} Q(\eta, \lambda) b_{j i} \Rightarrow k \geq j \\
& b_{k h} I(\eta, \lambda) b_{j i} \Rightarrow k=j \\
& k \geq j \Rightarrow b_{k h} S(\eta, \lambda) b_{j i}
\end{aligned}
$$

Note that Equations (5.a), (5.b), (5.c) correspond to the definition of a $(\sigma, \lambda)$-consistent set (Def. 4). Besides, (5.d) is related to the interpretation of $S$ as 'at least as good as'.

There is an inconsistency when for a pair $\left(b_{k h}, b_{j i}\right)$ one of the above implications is false. The number of inconsistencies with Eqs. (5) is obviously a function of $(\eta, \lambda)$.

In the following, we shall use the notation $N_{P}(\eta, \lambda), N_{Q}(\eta, \lambda), N_{I}(\eta, \lambda)$ and $N_{S}(\eta, \lambda)$ for the inconsistencies with Eqs. (5.a), (5.b), (5.c) and (5.d), respectively.

According to [10] the model's parameters should be inferred by solving the multiobjective optimization problem that follows: 
Minimize $\left\{N_{P}(\eta, \lambda), N_{Q}(\eta, \lambda), N_{I}(\eta, \lambda), N_{S}(\eta, \lambda)\right\}$

$(\eta, \lambda) \in f_{R}$

where $f_{R}$ denotes a feasible set. This set is determined by constraints that the $D M$ may impose on the model's parameters.

If $\sigma$ were calculated as in ELECTRE III or ELECTRE TRI (cf. [20]), with the simplification suggested in [18], the parameters in $\eta$ would be

i) the vector of weights $w_{i}$;

ii) the vector of indifference thresholds $q_{i}$;

iii) the vector of preference thresholds $p_{i}$;

iv) the vector of veto thresholds $v_{i}$;

v) the vector of discordance thresholds $u_{i}$.

The minimal set of constraints is: $w_{i}>0 ; 0 \leq q_{\mathrm{i}} \leq p_{i}<u_{i}<v_{i}(i=1, \ldots N) ; 0.5<\lambda \leq 1$.

The minimal set of constraints is: $w_{i}>0 ; 0 \leq q_{\mathrm{i}} \leq p_{i}<u_{i}<v_{i}(i=1, \ldots N) ; 0.5<\lambda \leq 1$.

Suppose that there is a parameter setting $\left(\eta_{0}, \lambda_{0}\right)$ satisfying $N_{P}\left(\eta_{0}, \lambda_{0}\right)=N_{Q}\left(\eta_{0}, \lambda_{0}\right)=N_{I}$ $\left(\eta_{0}, \lambda_{0}\right)=0$, and make $(\eta, \lambda)=\left(\eta_{0}, \lambda_{0}\right)$ in Eqs.(5). Upon comparing Eqs. (5) with Definition 4 , we conclude that the set $T$ is $(\sigma, \lambda)$ - consistent. That is, each $b \in T$ is $(T-\{b\}, \sigma, \lambda)$ consistently assigned according to Definition 3. So, the larger $T$, the more likely it is that $x \in U-T$ may be $(T, \sigma, \lambda)$-consistently assigned. The pair $\left(\sigma\left(\eta_{0}\right), \lambda_{0}\right)$ represents a decision policy that is consistent in the sense of Definition 1.

The following proposition is obvious:

Proposition 1: A set $T$ is $(\sigma, \lambda)$ - consistent for any pair $\left(\eta_{0}, \lambda_{0}\right)$ that fulfills $N_{P}\left(\eta_{0}, \lambda_{0}\right)=$ $N_{Q}\left(\eta_{0}, \lambda_{0}\right)=N_{I}\left(\eta_{0}, \lambda_{0}\right)=0$. Reciprocally, the condition $N_{P}\left(\eta_{0}, \lambda_{0}\right)=N_{Q}\left(\eta_{0}, \lambda_{0}\right)=N_{I}\left(\eta_{0}, \lambda_{0}\right)$ $=0$ is necessary for $(\sigma, \lambda)$ - consistency.

\section{Definition 5:}

Under the above notation a $(\sigma, \lambda)$ - consistent solution to Problem (6) on a set $T$ is defined as a pair $\left(\eta_{0}, \lambda_{0}\right)$ for which $N_{P}\left(\eta_{0}, \lambda_{0}\right)=N_{Q}\left(\eta_{0}, \lambda_{0}\right)=N_{I}\left(\eta_{0}, \lambda_{0}\right)=0$.

Equation (5.d) claims for consistency with $S(\eta, \lambda)$. However, false values of the implication in Equation (5.d) do not necessarily come from inconsistent preferences from the $D M$; rather, these inconsistencies come from incomparability or from a poor model of intensity of preference. Consequently, $N_{S}$ seems to be the less important objective in Problem (6). However, if $N_{S}$ were not appropriately controlled within acceptably low values, the credibility threshold would reach high values and the inconsistencies with Equations (5.a), (5.b) and (5.c) would tend to disappear; then $(\sigma, \lambda)$-consistency would be a consequence of a high level of incomparability.

$N_{P}$ seems to be the most important objective, followed by $N_{Q}$. However, a $P$ inconsistency that comes from $j-k=1$ in Eq. (5.a) may not be more significant than a $Q$ inconsistency coming from $j-k=2$ in Eq. (5.b), or than an $I$-inconsistency with $j-k=3$ in Eq. (5.c). Such effects of 'intensity of inconsistency' are not modelled by a simple count of $N_{P}$, $N_{Q}$ or $N_{I}$. Indeed, for this reason the THESEUS method adds inconsistencies only in two classes, $N_{1}$ and $N_{2}$. Hence, in order to increase the compatibility between the disaggregation analysis and THESEUS, instead of Problem (6) we suggest finding a best compromise of

Minimize ( $N_{\text {strong }}, N_{2 I}, N_{S}$ )

$(\eta, \lambda) \in f_{R}$

with $N_{\text {strong }}$ being the most important objective.

where:

$N_{\text {strong }}=N_{P}+N_{Q}+N_{11}$; 
$N_{1 I}, N_{2 I}$ count the first-order and second-order $I(\eta, \lambda)$-inconsistencies, respectively.

In case of several optimal solutions (in the objective space) for the first objective of Problem (7), all the solutions sharing that optimal value are compared by considering the other objective values (the second and third objective in Problem (7)). In this way, a best compromise may be identified.

A solution $(0,0, *)$ to Problem (7) corresponds to a $(\sigma, \lambda)$-consistent solution. If there is a Pareto solution $\left(0,0, N_{S}{ }^{*}\right)$ with an acceptably low value for $N_{S}{ }^{*}$, such a solution would be the best compromise solution of Problem (7). If there is no solution with these features, the $D M$ and the analyst should jointly explore the Pareto frontier of Problem (7), looking for an acceptable compromise solution even though this solution is not $(\sigma, \lambda)$-consistent.

Perhaps the easiest way of finding a compromise solution to Problem (7) consists in giving preemptive priority to $N_{\text {strong, }}$, and assigning the same importance to the remaining two objectives. This is equivalent to solving the problem

Minimize $\left(N_{\text {strong}}, N_{2 I}+N_{S}\right)$

$(\eta, \lambda) \in f_{R}$

with lexicographic priority favouring $N_{\text {strong. }}$.

As above, if there are several optimal solutions (in the objective space) for the first objective of Problem (8), all the solutions sharing that optimal value are compared by considering the second objective. So, the solution which reaches its minimum value is chosen. Problem (8) is a particular case of Problem (7) and it is not solved independently. Note that the solution to Problem (8) is a non-dominated solution of Problem (7) for which the sum $N_{2 I}+N_{S}$ is minimal. Once the Pareto frontier of Problem (7) has been obtained, the solution to Problem (8) is straightforward. Obtaining this solution does not require the $D M$ to express his/her preferences on the non-dominated solutions of Problem (7).

For solving Problem (7) and following [10], we use the Non-dominated Sorting Genetic Algorithm-II (NSGA-II) (e.g. [4]). In [10], Fernandez et al. solved Problem (6) with good results. Problem (7) is easier and basically the same algorithmic procedure can be used, probably with more effectiveness because only three objective functions need to be considered. In problems with two or three objective functions NSGA-II is one of the most efficient approaches in the literature on evolutionary multi-objective optimization (cf. [2]). This method ranks every member of a $K^{\prime}$-size population according to individual nondomination levels, applies evolutionary operators to build an offspring population, and combines parent and offspring populations in a new pool of size $2 K^{\prime}$. This combined population is sorted into non-dominated classes. The next $K$ '-size population is obtained by selecting the best individuals of the parent-offspring combined pool. In order to keep diversity, a crowding distance (a density estimator) is associated with every individual.

For the selection of 'parents', NSGA-II uses a special kind of binary tournament called the 'crowded tournament selection operator' ([4]). It works as follows: let $i, j$ be two randomly selected solutions from the parent population. Solution $i$ wins the tournament over $j$ whenever one of the following conditions is true:

1) if solution $i$ has a better rank than $j$;

2) if solutions $i$ and $j$ have the same rank but solution $i$ has a better crowding distance than $j$ (that is, the crowding distance associated with $i$ is greater than that associated with $j$ ). 
Point 1 ensures that the winner lies on a better non-dominated front. Point 2 solves possible ties between solutions on the same front by making a decision according to crowding distances. In this case, the winner resides in a less crowded region. 1.

Individuals are represented by a string composed of $5 n+1$ positions as shown in Figure

Figure 1: Individual coding

\begin{tabular}{|l|l|l|l|l|l|l|l|l|l|l|l|l|l|l|l|l|}
\hline$p_{1}$ & $u_{1}$ & $v_{1}$ & $\cdots$ & $\cdots$ & $p_{n}$ & $u_{n}$ & $v_{n}$ & $w_{1}$ & $w_{2}$ & $\cdots$ & $w_{n}$ & $q_{1}$ & $q_{2}$ & $\cdots$ & $q_{n}$ & $\lambda$ \\
\hline
\end{tabular}

Different individuals are considered as different solutions in each NSGA-II population, so several individuals can be pre-image of the same point in the objective space thus sharing the same 'fitness'.

For the weights, we use the approach in [1]. $n-1$ uniform random numbers are generated in $(0 ; 1)$; further, these are ranked $0<a_{1}<a_{2} \ldots<a_{n-1}<1$, and the weights are calculated as $w_{\mathrm{i}}$ $=a_{i}-a_{i-1}$. Thus, the normalization condition $\left(w_{1}+w_{2}+\ldots+w_{n}=1\right)$ is satisfied and the random weights are uniformly distributed.

One-point crossover is used (cf. [16]). $2 n+1$ possible crossover points are defined on the individual (see Figure 2). Given the two parents, the specific crossover point is randomly generated. A repair process is used to keep the individual within the feasible region.

Figure 2: Possible crossover points

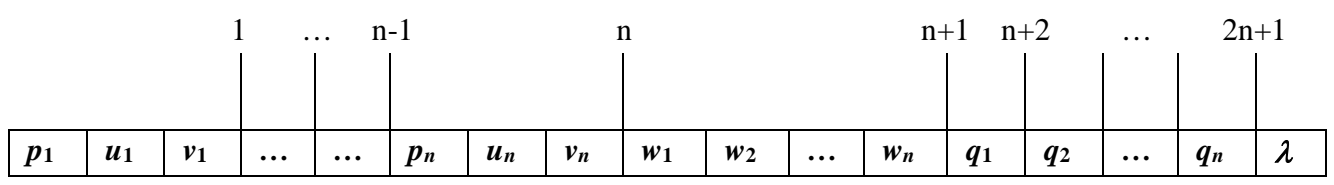

The Uniform mutation is used here as in [16]. The parameters of the evolutionary search are set to: Number of generations $=1000$, Population size $=100$, Crossover probability $=$ 0.8 , Mutation probability $=0.05$. More details can be found in [10].

\subsection{Finding the best parameter setting in a THESEUS frame}

Let us denote by $\left\{\boldsymbol{P}^{*}\right\}_{z^{*}}$ the set of points $(\eta, \lambda)$ in the parameter space that are the pre-image of a known Pareto solution $z^{*}$ in the objective space of Problem (7). The following steps are necessary to set THESEUS:

Step 1: Set the constraints $f_{R}$;

Step 2: Given a reference set $T$, apply the evolutionary algorithm described in Section 3.1 to find an approximation to the Pareto frontier of Problem (7); let us denote such a set by $Z$ (the known Pareto frontier).

Step 3: If there is a $(\sigma, \lambda)$ - consistent solution with an acceptable low value of $N_{S}$, then choose this as the best compromise $z_{\text {best }}$; obtain the most central point $(\eta, \lambda)_{\text {best }}$ in the set $\left\{\boldsymbol{p}^{*}\right\} z_{\text {best }}$ if there is more than one.

Else: 
Look in $Z$ for a good compromise solution $z_{\text {good. }}$. If the $D M$ is not available or if (s)he is averse to comparing solutions in $Z$, then choose the solution to Problem (8) as the compromise solution $z_{\text {easy. }}$.

Obtain the most central point $(\eta, \lambda)_{\text {easy }}$ in the set $\left\{\boldsymbol{p}^{*}\right\} z_{\text {easy }}$ if there is more than one.

\section{Remarks:}

a) The most central points $(\eta, \lambda)_{\text {best }}$ and $(\eta, \lambda)_{\text {easy }}$ are the nearest points (in the sense of a normalized Euclidean distance) to the centroids of the sets $\left\{\boldsymbol{p}^{*}\right\} z_{\text {best }}$ and $\left\{\boldsymbol{p}^{*}\right\} z_{\text {easy }}$.

The exploration in $Z$ may use a set $B \subset U, B \cap T=\Phi$ of objects whose assignments are known; those assignments may be compared with the assignments suggested by points in $\left\{\boldsymbol{p}^{*}\right\}_{z^{*}}$, helping to reach the best compromise setting. Once this setting has been found, THESEUS is ready to suggest sorting decisions.

\section{Extensive computer experimentation}

Concerning the solutions provided by the combined PDA-THESEUS approach, let us revisit the questions that we have formulated in the the introduction:

A) To what extent is their quality affected by the number of categories and criteria?

B) To what extent is their quality influenced by the cardinality of the reference set?

C) To what extent is the quality of solutions influenced by the DM's level of involvement in a co-constructive process with the decision-analyst?

D) How far can the quality of the final solution be improved by a comparison process between different potential solutions?

A formal theoretical answer to the above questions is impossible. Therefore, we choose to perform an experimental analysis in which a great diversity of decision-maker preferences is simulated and many reference sets are randomly built. The influence of imposing constraints on the model's parameters is examined. The quality of the solution to Problem (8) (a solution that is independent of the decision-maker's preferences) is compared with that of other solutions that require the $D M$ to express preferences on $Z$. Thus, the combined PDA-THESEUS performance is explored for a wide range of simulated decision-maker preferences (expressed by the model's parameters), and different levels of involvement from the decision-maker, numbers of categories, numbers of criteria, and cardinalities of the reference set.

We shall analyse the performance of three solutions in the parameter space:

$(\eta, \lambda)_{\text {easy: }}$ the point corresponding to the solution to Problem (8); this point can be detected automatically, being independent of the $D M$ 's preferences on $Z$. No effort from the $D M$ is therefore needed in order to identify this point.

$(\eta, \lambda)_{\text {Eucl }}$ : the nearest point (in the sense of a normalized Euclidean distance) to the parameter setting used in the simulation study;

$(\eta, \lambda)_{\text {Tche }}$ : the nearest point (in the sense of a normalized Tchebychev distance) to the parameter setting used in the simulation study.

$(\eta, \lambda)_{\text {Eucl }}$ and $(\eta, \lambda)_{\text {Tche }}$ belong to the pre-image of the known Pareto frontier of Problem (7).

The main design factors of the experiment are shown in Table 1. 
Table 1. Summary of experimental design factors

\begin{tabular}{|l|l|}
\hline \multicolumn{1}{|c|}{ Factor } & \multicolumn{1}{c|}{ Levels } \\
\hline Number of criteria $(N)$ & $3,5,7,9$ \\
\hline Number of categories $(M)$ & $3,5,7,9$ \\
\hline Number of reference objects (card $(T)$ ) & $50,100,200$ \\
\hline Sample size in the model's parameter space & 50 \\
\hline Size of validation samples & 1000 \\
\hline $\begin{array}{l}\text { Number of runs of the evolutionary } \\
\text { algorithm }\end{array}$ & 20 \\
\hline Constraint settings & $\begin{array}{c}\text { 'minimal', 'additional' } \\
\text { (see remarks below) }\end{array}$ \\
\hline
\end{tabular}

The experiment involves the following steps:

- Generate the outranking model's parameter set. This set of parameter values, which is used in the simulation study, is denoted by $(\eta, \lambda)_{\text {sim }}$.

- Construct a $(\sigma, \lambda)$-consistent reference set $T$ (Def. 4) with the desired cardinality; $\sigma$ is calculated as in ELECTRE III with the simplification of the discordance index proposed in [18].

- Once $T$ has been built, solve Problems (7) and (8) running 20 times the algorithm described in Sections 3.1 and 3.2. Find $(\eta, \lambda)_{\text {easy }},(\eta, \lambda)_{\text {Eucl }}$ and $(\eta, \lambda)_{\text {Tche. }}$

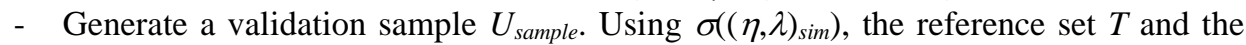
THESEUS assignment rule, assign each object $x$ of $U_{\text {sample }}$ to a category $C_{\text {sim }}(x)$ or to an interval $\left[C_{L}, C_{H}\right]_{\text {sim. }}$. Do the same using $(\eta, \lambda)_{\text {easy }},(\eta, \lambda)_{\text {Eucl }}$ and $(\eta, \lambda)_{\text {Tche }}$ and count the full coincidences with $(\eta, \lambda)_{\operatorname{sim}}$. A full coincidence (in the following simply 'a coincidence') happens if and only if $C_{\operatorname{sim}}(x)=C_{\eta \lambda}(x)$ or $\left[C_{L}, C_{H}\right]_{\operatorname{sim}}=\left[C_{L}, C_{H}\right]_{\eta \lambda}$.

\section{Remarks:}

1. In solving Problems (7) and (8) the constraints are set in two different and independent ways: i) under a minimal set of constraints that concern the natural values of the model's parameters; and ii) by imposing constraints that can reflect certain beliefs from the $D M$ related to acceptable parameter values.

2. Since the model's parameters have a specific interpretation, the random generation is performed in a way that keeps their relationships and their semantic meaning ([12]). Veto power is not conceded to criteria having weights clearly lower than the average. As the $i$-th criterion has veto capacity, its pre-veto threshold $u_{i}$ is generated from a normal distribution centred in the middle value of the interval $\left[p_{i}, v_{i}\right]$. Conditions $0 \leq q_{i} \leq p_{i}<u_{i}<v_{i}(i=1, \ldots N)$ are imposed. This setting is called 'minimal constraints'.

3. $D M$ beliefs on acceptable parameter values are simulated by restricting them to $(\eta, \lambda)_{\operatorname{sim}}-1 / 3(\eta, \lambda)_{\operatorname{sim}} \leq(\eta, \lambda) \leq(\eta, \lambda)_{\operatorname{sim}}+1 / 3(\eta, \lambda)_{\operatorname{sim}}$. This setting is called 'additional constraints'.

4. For achieving coincidence with the assignments from $(\eta, \lambda) *$, it would be sufficient that $S(\eta, \lambda)_{\operatorname{sim}}=S(\eta, \lambda) *$. The closer $S(\eta, \lambda) *$ is to $S(\eta, \lambda)_{\text {sim }}$, the higher should be the number of coincidences. So, the more similar $(\eta, \lambda)$ is to $(\eta, \lambda)_{\operatorname{sim}}$, the more 
coincidences $C_{\operatorname{sim}}(x)=C_{\eta \lambda}(x)$ or $\left[C_{L}, C_{H}\right]_{\operatorname{sim}}=\left[C_{L}, C_{H}\right]_{\eta \lambda}$ there will be. Thus, the number of coincidences is a measure of the quality of the solutions of Problems (7) and (8).

5. The prior probability of a random coincidence is $2 /(M .(M-1)+2 M)$.

Some results are shown in Figures 3-8 in terms of the average rate of coincidences $r_{c}$ when $(\eta, \lambda)=(\eta, \lambda)_{\text {easy. }}$. The closer $r_{c}$ is to 1 , better $(\eta, \lambda)_{\text {easy }}$ reflects the simulated $D M$ with parameters $(\eta, \lambda)_{\text {sim. }}$. Figures 3-5 correspond to the case in which no constraints of the type described in the third remark are imposed (the $D M$ is not involved in setting the feasible region of Problems (7) and (8)). Figures 6-8 provide some results obtained under the constraints expressed in the third remark above.

Figure 3: Average rate of coincidences vs $\operatorname{card}(T)$ (minimal constraints)

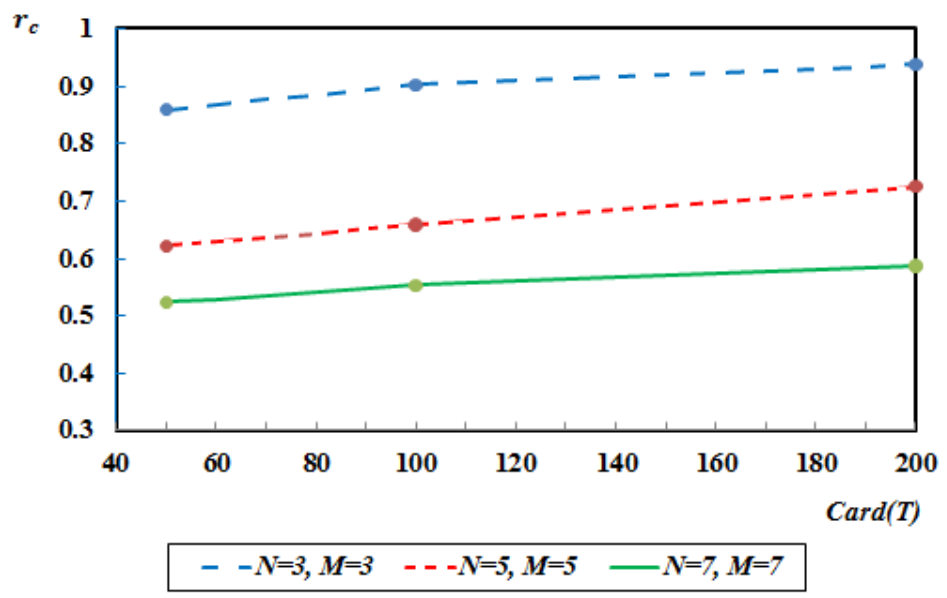

Figure 4: Average rate of coincidences vs $N$ with $M=3$ and minimal constraints

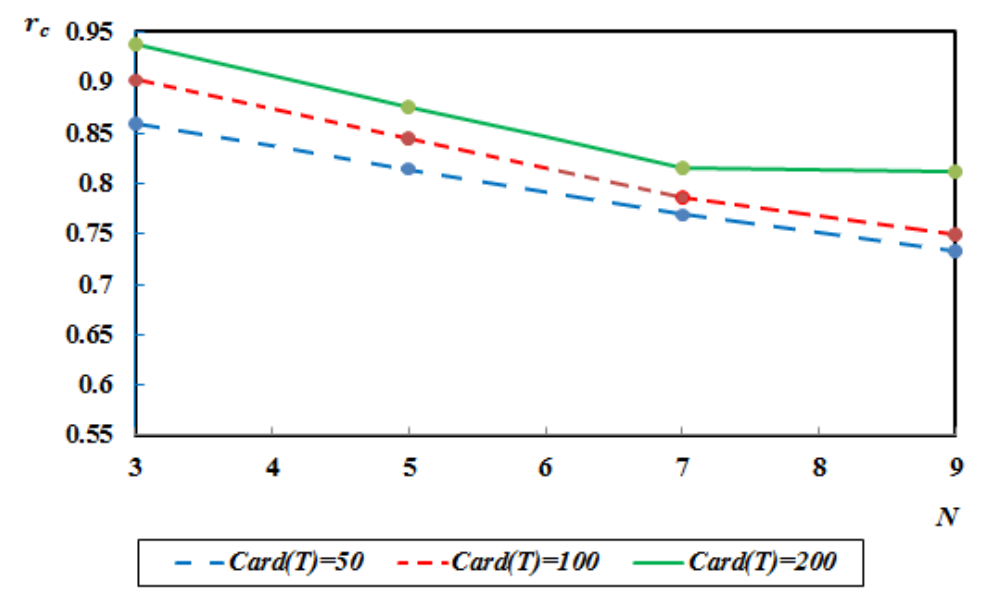


Figure 5: Average rate of coincidences vs $M$ with $N=3$ and minimal constraints

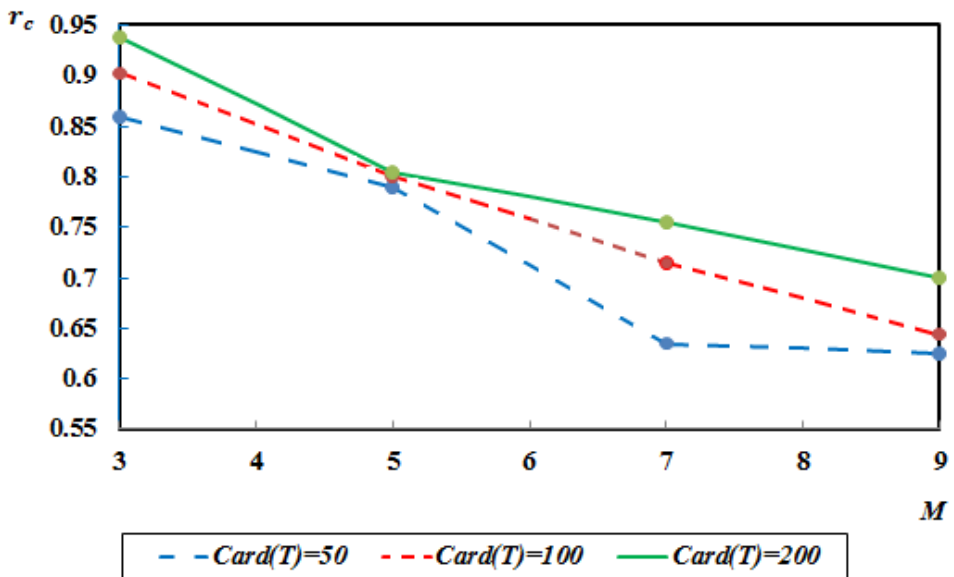

Figure 6: Average rate of coincidences vs $\operatorname{card}(T)$ and additional constraints

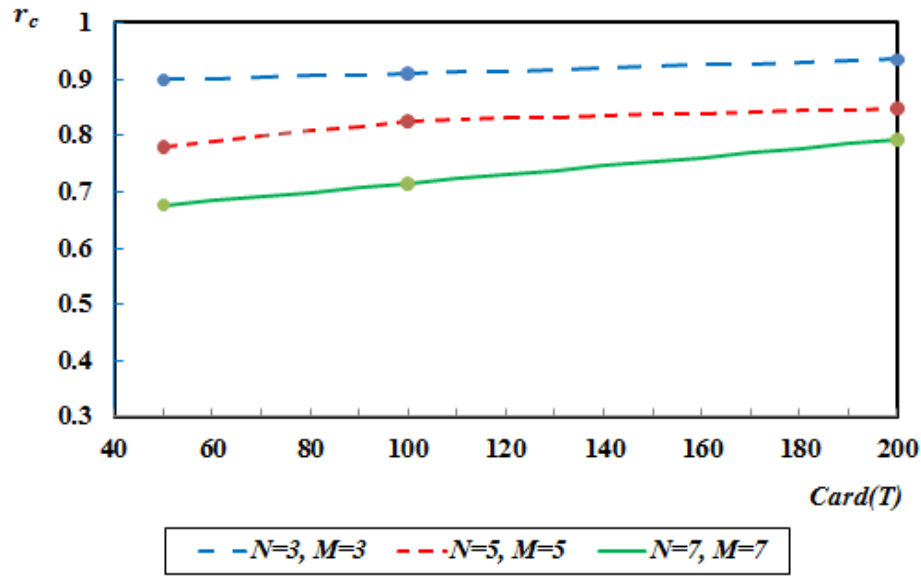


Figure 7: Average rate of coincidences vs $N$ with $M=3$ and additional constraints

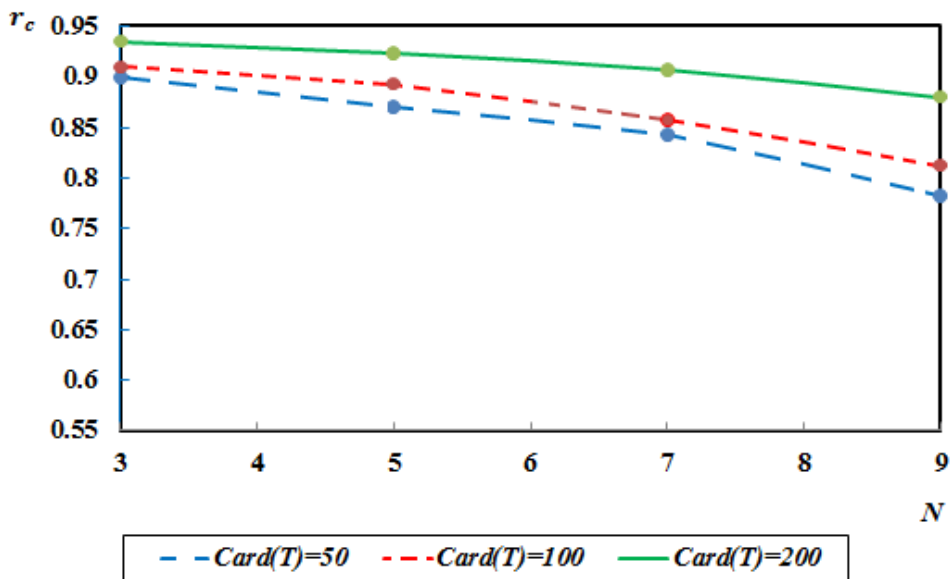

Figure 8: Average rate of coincidences vs $M$ with $N=3$ and additional constraints

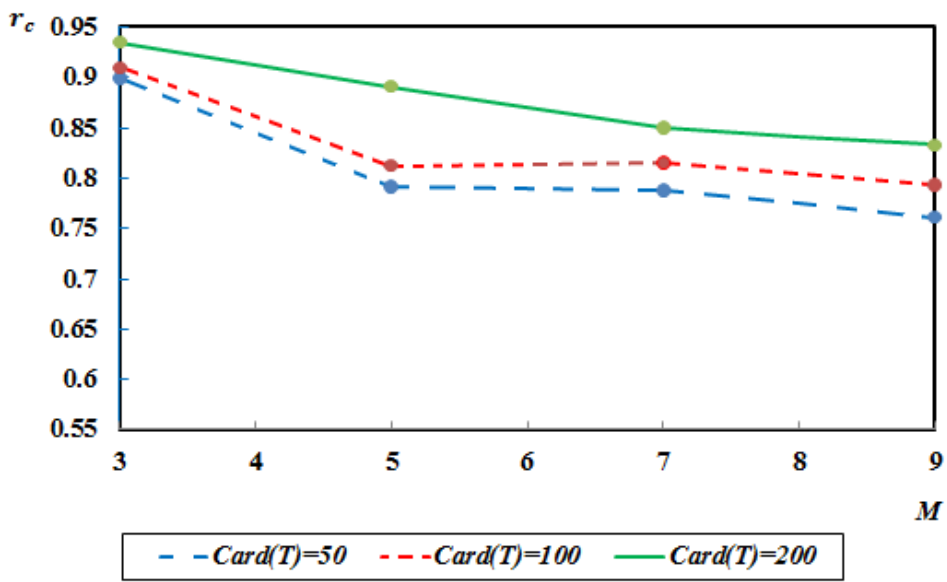

The detailed results are provided in Tables 3 and 4 (see the Appendix) in terms of the average number of coincidences with $(\eta, \lambda)_{\text {sim. }}$. This number is counted on a validation sample with 1000 elements. Since $(\eta, \lambda)_{\text {easy }}$ can be automatically detected, no involvement of the DM is reflected in Figures 3-5 and the column 'Average coincidences with $(\eta, \lambda)_{\text {easy }}$ ' of Table 3 (see the Appendix). Besides, Figures 6-8 and the column 'Average coincidences with $(\eta, \lambda)_{\text {easy' }}$ of Table 4 (see the Appendix) correspond to the case in which the $D M$ is able to express his/her beliefs and setting some constraints on the parameter values, but (s)he is not willing to compare points on the Pareto frontier of Problem (7).

Table 2 shows a comparison between two levels of $D M$ involvement. Its column ' $D M$ is not involved' coincides with the column 'Average coincidences with $(\eta, \lambda)_{\text {easy' }}$ in Table 3 (see the Appendix). The column ' $D M$ is involved' in Table 2 contains the best values of 
the coincidences that an involved $D M$ could identify with a deep analysis and comparison of points on the Pareto frontier of Problem (7) (see Table 4 in the Appendix). Normality of the data was checked by the Anderson-Darling test, and statistical significance was analysed by using a two sample t-test. Let $\mu_{n i}$ be the population mean of coincidences without $D M$ involvement. Let $\mu_{i}$ denote the population mean with involvement. The null hypothesis was $\mu_{i} \leq \mu_{n i}$. The alternative hypothesis was $\mu_{i}-\mu_{n i}>0$. The significance level was set to 0.05 . Table 2 only points out the experimental points for which the null hypothesis was rejected.

Table 2. Average number of coincidences with $(\eta, \lambda)_{s i m}$ in two levels of $D M$ involvement

\begin{tabular}{|c|c|c|c|c|c|}
\hline$M$ & $N$ & $\operatorname{card}(T)$ & $D M$ is not involved & $D M$ is involved & \% of improvement \\
\hline \multirow[t]{5}{*}{3} & 7 & 100 & 786.4 & 881.1 & 12.0 \\
\hline & & 200 & 815.5 & 915.8 & 12.3 \\
\hline & 9 & 50 & 732.0 & 820.9 & 12.1 \\
\hline & & 100 & 749.1 & 844.4 & 12.7 \\
\hline & & 200 & 812.0 & 908.9 & 11.9 \\
\hline \multirow[t]{12}{*}{5} & 3 & 50 & 790.0 & 886.6 & 12.2 \\
\hline & & 100 & 801.4 & 902.7 & 12.6 \\
\hline & & 200 & 804.9 & 928.2 & 15.3 \\
\hline & 5 & 50 & 621.7 & 818.3 & 31.6 \\
\hline & & 100 & 659.9 & 872.0 & 32.1 \\
\hline & & 200 & 725.3 & 899.7 & 20.0 \\
\hline & 7 & 50 & 630.1 & 750.2 & 19.1 \\
\hline & & 100 & 629.2 & 831.9 & 32.2 \\
\hline & & 200 & 676.9 & 867.0 & 28.1 \\
\hline & 9 & 50 & 534.1 & 751.6 & 40.7 \\
\hline & & 100 & 547.9 & 808.2 & 47.5 \\
\hline & & 200 & 554.9 & 839.1 & 51.2 \\
\hline \multirow[t]{12}{*}{7} & 3 & 50 & 635.1 & 873.7 & 37.6 \\
\hline & & 100 & 714.4 & 897.8 & 25.7 \\
\hline & & 200 & 754.8 & 922.2 & 22.2 \\
\hline & 5 & 50 & 542.2 & 801.8 & 47.0 \\
\hline & & 100 & 546.3 & 859.6 & 57.3 \\
\hline & & 200 & 599.9 & 894.4 & 49.1 \\
\hline & 7 & 50 & 523.5 & 720.3 & 37.6 \\
\hline & & 100 & 552.6 & 796.4 & 44.1 \\
\hline & & 200 & 586.7 & 838.0 & 42.8 \\
\hline & 9 & 50 & 475.7 & 669.1 & 40.7 \\
\hline & & 100 & 531.9 & 730.1 & 37.3 \\
\hline & & 200 & 540.7 & 802.2 & 48.4 \\
\hline \multirow[t]{8}{*}{9} & 3 & 50 & 625.5 & 843.2 & 34.8 \\
\hline & & 100 & 643.6 & 870.1 & 35.2 \\
\hline & & 200 & 700.1 & 914.6 & 30.6 \\
\hline & 5 & 50 & 469.9 & 792.6 & 68.7 \\
\hline & & 100 & 551.1 & 846.6 & 53.5 \\
\hline & & 200 & 582.9 & 889.2 & 52.5 \\
\hline & 7 & 50 & 417.5 & 678.1 & 62.4 \\
\hline & & 100 & 544.1 & 774.4 & 42.3 \\
\hline
\end{tabular}




\begin{tabular}{|l|c|c|c|c|c|}
\hline & & 200 & 539.3 & 810.9 & 50.4 \\
\hline & 9 & 50 & 420.2 & 638.8 & 52.0 \\
\hline & & 100 & 444.5 & 707.1 & 59.1 \\
\hline & & 200 & 498.1 & 769.6 & 54.5 \\
\hline
\end{tabular}

\section{Remarks:}

1. The quality of solutions (measured by the number of coincidences with $(\eta, \lambda)_{\text {sim }}$ ) is better when additional constraints are imposed (compare Figures 6-8 with Figures 3-5). This suggests that the PDA-THESEUS combined proposal may be improved when the $D M$ is willing to provide his/her beliefs on acceptable parameter values.

2. Fifteen times under the minimal constraints (see the column 'Better than $(\eta, \lambda)_{\text {easy }}$, Table 3 in the Appendix) and twenty times under the additional constraints (see the column 'Better than $(\eta, \lambda)_{\text {easy }}$ ', Table 4 in the Appendix), there is at least one point in $Z$ for which the number of coincidences with $(\eta, \lambda)_{\operatorname{sim}}$ is significantly greater than the one obtained by $(\eta, \lambda)_{\text {easy. }}$ This underlines the importance of the DM's active participation in exploring the set $Z$ in a co-constructive process with the decision analyst.

3. $(\eta, \lambda)_{\text {Eucl }}$ and $(\eta, \lambda)_{\text {Tche }}$ cannot be used in practice because the analyst-DM couple do not know the true parameter values. But when we proved that $(\eta, \lambda)_{\text {Eucl }}$ and/or $(\eta, \lambda)_{\text {Tche }}$ are significantly better than $(\eta, \lambda)_{\text {easy }}$, one thing is clear: there is at least one parameter setting as the Pareto optimal point of Problem (7) that outperforms $(\eta, \lambda)_{\text {easy. }}$ This is an indication for the analyst-DM couple. Comparing the results shown in columns 4-7 (Tables 3 and 4 in the Appendix), the analyst-DM can choose between accepting $(\eta, \lambda)_{\text {easy }}$ and finding a better solution by exploring other Pareto optimal points in the parameter space. Note that the best result between $(\eta, \lambda)_{\text {Eucl }}$ and $(\eta, \lambda)_{\text {Tche }}$ may be outperformed by other Pareto optimal points. Therefore, the values obtained from $(\eta, \lambda)_{\text {Eucl }}$ and $(\eta, \lambda)_{\text {Tche }}$ are the lower bounds of others that can be found in the pre-image of $Z$. The exploration of the Pareto set may be performed by testing different potential settings with assignments not belonging to the original reference set.

4. Generalizing the above remarks, Table 2 shows the importance of the DM's active role in a co-constructive process with the decision analyst.

5. Comparing the first and second halves of Tables 3 and 4 (see the Appendix), $(\eta, \lambda)_{\text {easy }}$ provides good results in sorting problems with few categories; with an increase in $M$ up to five, $(\eta, \lambda)_{\text {Eucl }}$ and $(\eta, \lambda)_{\text {Tche }}$ tend to perform better.

6 . In general, the quality of the solutions is degraded by the increment in $M$ and $N$ (see, for instance, Figures 4, 5, 7, and 8). However, values of $60 \%$ of coincidences with $(\eta, \lambda)_{\text {sim }}$ should not be judged as bad if we consider that with $M=9$ the prior probability of a random coincidence with $C_{\text {sim }}(x)$ or $\left[C_{L}, C_{H}\right]_{\operatorname{sim}}$ is $1 / 45$.

7. The quality of solutions seems to be improved by the increments in the cardinality of the reference set (see, for instance, Figures 3 and 6); such dependence is very often statistically significant when card $(T)=200$ is compared with card $(T)=50$.

The last remarks could be explained by the following arguments:

The assignment of $x$ to $C_{s i m}(x)=C_{k^{*}}$ has to be supported by reference objects $b_{k^{*}}$ fulfilling conditions of the THESEUS Uniqueness Theorem in Section 2. The assignment of $x$ to $\left[C_{L}, C_{H}\right]_{\operatorname{sim}}$ should be supported by reference objects $b_{L n}$ and $b_{H j}$ such that $x S(\eta, \lambda)_{\operatorname{sim}} b_{L n}$ and $b_{H j} S(\eta, \lambda)_{\operatorname{sim}} x$. Reference actions fulfilling those conditions are pieces of 
information that support assignments. The cardinalities of $S(\eta, \lambda)_{\operatorname{sim}}$ and $S(\eta, \lambda) *$ are reduced when $N$ increases because of incomparability effects. Also, when $M$ increases and card $(T)$ decreases, the number of pieces of information that support particular assignments reduces too. With very few pieces of information supporting $C_{\text {sim }}$ or $\left[C_{L}, C_{H}\right]_{\text {sim }}$, a slight difference between $(\eta, \lambda) *$ and $(\eta, \lambda)_{\text {sim }}$ may provoke non-coincident assignments.

\section{Concluding remarks}

The THESEUS multi-criteria sorting method suggests assignments that are as compatible as possible with the previous assignments in the reference set and the fuzzy outranking model $\sigma$. Consistency requires that the assignments of the reference actions should be compatible with $\sigma$-values. The model of the crisp outranking relation derived from $\sigma$ should be as close as possible to the $D M$ 's preference relation 'at least as good as'.

THESEUS does not perform well if the $\sigma$-model's parameters are directly set by the $D M$. Hence, we have proposed here a combined approach, in which the preferencedisaggregation evolutionary method in [10] is adapted to the THESEUS framework. That evolutionary multi-objective-based method works by minimizing certain counts of inconsistencies, which is similar to the way in which THESEUS makes assignment decisions.

The evolutionary algorithm is (in structure) not essentially different from those described by our previous paper (cf. [10]). Here, the novelty consists in:

First: THESEUS is extended in order to become a multi-criteria PDA-sorting method. We propose a framework with the conjoint use of the evolutionary multi-objective optimization based on PDA and THESEUS. The concept of $(\sigma, \lambda)$-consistency is introduced and the multi-objective problem is adapted to THESEUS (see discussion in Sections 3.1 and 3.2).

Second and more important: to a certain extent, this paper finds answers to the fundamental questions about the quality of solutions, and finds how this quality is influenced by the characteristics of the data and by the level of involvement of the $D M$.

From an extensive experiment (during which many models of preferences and sizes of problems, and different degrees of decision maker involvement were explored), we have obtained the following conclusions:

I. The PDA-THESEUS combined proposal performs very well in sorting problems with few categories and criteria; although acceptable, its performance is degraded by the increments in the number of categories and criteria; it tends to improve when the cardinality of the reference set increases.

II. The proposal may perform better if the $D M$ is willing to provide his/her beliefs on acceptable parameter values.

III. When the number of categories $(M)$ is less than five, the $D M$ may accept $(\eta, \lambda)_{\text {easy }}$ (the set of parameters generated automatically by our proposal) as the best setting. If $M \geq 5$ the quality of solutions improves when the $D M$ is involved in the process of comparing and evaluating different solutions on the known Pareto frontier of the multi-objective parameter elicitation problem. Analysing the results shown in columns 4-7 of Tables 3 and 4 in the Appendix, the analyst-DM can choose between accepting $(\eta, \lambda)_{\text {easy }}$ and finding a better solution by exploring other Pareto optimal 
points in the parameter space. The exploration of the Pareto set may be performed by testing different potential settings with assignments not belonging to the original reference set.

IV. The greater the number of categories, the more important the involvement of the $D M$ in relation to the above points.

Our results have proved the importance of the DM's role in comparing different points on the approximated Pareto frontier of Problem (7). Analyses of the ways in which the $D M$ 's involvement might take place will be the topic of future research. It is interesting to examine which constraints on the parameters add most to the improvement of the results. Also, a study of the effect of noisy information that the $D M$ may provide would be welcome.

\section{Acknowledgements}

We acknowledge the support from CONACYT project no. 236154. We also thank the anonymous reviewers for their helpful remarks.

\section{References}

[1] Butler, J., Jia, J., Dyer, J. (1997): Simulation techniques for the sensitivity analysis of multi-criteria decision models, European Journal of Operational Research 103, 3, 531546.

[2] Coello, C.A., Lamont, G.B., Van Veldhuizen, D.A. (2007): Evolutionary Algorithms for Solving Multi_Objective Problems, Second Edition. Springer, New York.

[3] Corrente, S., Doumpos, M., Greco, S., Slowinski, R., Zopounidis, C. (2015): Multiple criteria hierarchy process for sorting problems based on ordinal regression with additive value functions, Annals of Operations Research DOI 10.1007/s10479-0151898-1.

[4] Deb, K. (2001): Multi-Objective Optimization using Evolutionary Algorithms, John Wiley \& Sons, Chichester-New York-Weinheim-Brisbane-Singapore-Toronto.

[5] Dias, L., Mousseau, V., Figueira J., Climaco, J. (2002): An aggregation/disaggregation approach to obtain robust conclusions with ELECTRE-TRI, European Journal of Operational Research, 138, 2, 332-348.

[6] Doumpos, M., Marinakis, Y., Marimaki, M., Zopounidis, C. (2009): An evolutionary approach to construction of outranking models for multicriteria classification: The case of ELECTRE TRI method, European Journal of Operational Research, 199, 2,496505.

[7] Doumpos, M., Zopounidis, C., Galariotis, E. (2014): Inferring robust decision models in multicriteria classification problems: An experimental analysis, European Journal of Operational Research, 236, 2, 601-611.

[8] Fernandez, E., Navarro, J., Bernal, S. (2009): Multicriteria sorting using a valued indifference relation under a preference disaggregation paradigm, European Journal of Operational Research, 198, 2, 602-609.

[9] Fernandez, E., Navarro, J. (2011): A new approach to multicriteria sorting problems based on fuzzy outranking relations: The THESEUS method, European Journal of Operational Research, 213, 2, 405-413. 
[10] Fernandez E., Navarro J., Mazcorro G.(2012): Evolutionary multi-objective optimization for inferring outranking model's parameters under scarce reference information and effects of reinforced preference, Foundations of Computing and Decision Sciences, 37, 3, 163-197.

[11] Fernandez E., Navarro J., Salomon E. (2014): Automatic enhancement of the reference set for multi-criteria sorting in the frame of THESEUS method, Foundations of Computing and Decision Sciences 39, 2, 57-77.

[12] Fernandez E., Navarro J., Covantes, E., Rodriguez, J. (2016): Analysis of the effectiveness of the THESEUS multi-criteria sorting method: theoretical remarks and experimental evidence, TOP, DOI: 10.1007/s11750-016-0433-0.

[13] Greco, S., Mousseau, V., Slowinski, R. (2008): Ordinal regression revisited: Multiple criteria ranking with a set of additive value functions, European Journal of Operational Research, 191, 415-435.

[14] Jacquet-Lagreze, E., Siskos, J. (1982): Assessing a set of additive utility functions for multicriteria decision making: The UTA method, European Journal of Operational Research, 10, 2, 151-164.

[15] Köksalan, M, Mousseau, V, Özpeynirci, Ö, Özpeynirci, SB. (2009): A New Outranking-Based Approach for Assigning Alternatives to Ordered Classes, Naval Research Logistics, 56, 1, 74-85.

[16] Michalewicz, Z. (1996): Genetic Algorithms + Data Structures = Evolution Programs, Springer Verlag, Berlin-Heidelberg-New York.

[17] Mousseau, V., Slowinski, R.(1998): Inferring an ELECTRE-TRI model from assignment examples, Journal of Global Optimization, 12, 2, 157-174.

[18] Mousseau, V., Dias, L.C. (2004): Valued outranking relations in ELECTRE providing manageable disaggregation procedures, European Journal of Operational Research, 156, 2, 467-482.

[19] Rocha, C, Dias, L.C. (2008): An algorithm for ordinal sorting based on ELECTRE with categories defined by examples, Journal of Global Optimization, 42, 2, 255-277.

[20] Roy, B. (1990): The outranking approach and the foundations of ELECTRE methods, in: Bana e Costa, C.A., (ed.), Reading in Multiple Criteria Decision Aid, SpringerVerlag, Berlin, 155-183.

[21] Roy B. (1996): Multicriteria methodology for Decision Aiding, Kluwer Academic Publisher, Dordrecht-Boston-London.

[22] Roy, B., Slowinski, R. (2008): Handling effects of reinforced preference and counterveto in credibility of outranking, European Journal of Operational Research, 188, 1, 185-190.

[23] Spyridakos, A., Yannacopoulos, D. (2015): Incorporating collective functions to multicriteria disaggregation-aggregation approaches for small group decision making, Annals of Operations Research, 227, 1, 119-136.

[24] Vetschera, R., Chen, Y., Hipel, K.W. (2010): Robustness and information levels in case-based multiple criteria sorting, European Journal of Operational Research, 202, 3, 841-852.

Received 26.01.2016, Accepted 19.09.2016 


\section{APPENDIX}

The results are shown in Tables 3 and 4 . The column 'Better than $(\eta, \lambda)_{\text {easy' }}$ takes into account statistical significance.

This analysis was performed by using paired sample t-tests. The null hypotheses were $\mu_{\text {(Eucl-easy) }} \leq 0$ and $\mu_{\text {(Tche-easy) }} \leq 0$. The alternative hypotheses were $\mu_{\text {(Eucl-easy) }}>0$ and $\mu_{\text {(Tche-easy) }}$ $>0$, respectively.

Table 3. Average number of coincidences with $(\eta, \lambda) \operatorname{sim}$ (minimal constraints)

\begin{tabular}{|c|c|c|c|c|c|c|}
\hline$M$ & $N$ & card $(T)$ & $\begin{array}{c}\text { Ave. } \\
\text { coincidences } \\
(\eta, \lambda)_{\text {easy }}\end{array}$ & $\begin{array}{c}\text { Ave. } \\
\text { coincidences } \\
(\eta, \lambda)_{\text {Eucl }}\end{array}$ & $\begin{array}{c}\text { Ave. } \\
\text { coincidences } \\
(\eta, \lambda)_{\text {Tche }}\end{array}$ & $\begin{array}{c}\text { Better than } \\
(\eta, \lambda)_{\text {easy }}{ }^{*}\end{array}$ \\
\hline 3 & 3 & 50 & 859.4 & 843.5 & 801.9 & - \\
\hline & & 100 & 902.4 & 899.2 & 891.0 & - \\
\hline & & 200 & 938.7 & 935.3 & 895.1 & - \\
\hline & 5 & 50 & 814.0 & 801.7 & 797.3 & - \\
\hline & & 100 & 844.2 & 830.2 & 819.8 & - \\
\hline & & 200 & 876.6 & 860.0 & 810.0 & - \\
\hline & 7 & 50 & 768.8 & 758.7 & 765.4 & - \\
\hline & & 100 & 786.4 & 801.8 & 794.6 & - \\
\hline & 9 & 200 & 815.5 & 822.4 & 804.1 & - \\
\hline & & 100 & 732.0 & 729.9 & 728.4 & - \\
\hline & & 200 & 812.0 & 760.3 & 721.2 & - \\
\hline 5 & 3 & 50 & 790.0 & 805.0 & 808.6 & - \\
\hline & & 100 & 801.4 & 832.1 & 807.9 & - \\
\hline & & 200 & 804.9 & 846.4 & 839.3 & - \\
\hline & 5 & 50 & 621.7 & 736.0 & 727.4 & $(\eta, \lambda)_{\text {Eucl }}$ \\
& & & & & & $(\eta, \lambda)_{\text {Tche }}$ \\
\hline & & 100 & 659.9 & 762.0 & 704.1 & $(\eta, \lambda)_{\text {Eucl }}$ \\
\hline & & 200 & 725.3 & 789.5 & 757.9 & - \\
\hline & 7 & 50 & 630.1 & 671.2 & 667.6 & - \\
\hline & & 100 & 629.2 & 737.2 & 693.5 & $(\eta, \lambda)_{\text {Eucl }}$ \\
\hline & & 200 & 676.9 & 775.4 & 710.9 & $(\eta, \lambda)_{\text {Eucl }}$ \\
\hline & 9 & 50 & 534.1 & 589.6 & 546.9 & - \\
\hline & & 100 & 547.9 & 601.9 & 598.0 & - \\
\hline & & 200 & 554.9 & 642.5 & 603.2 & $(\eta, \lambda)_{\text {Eucl }}$ \\
\hline 7 & 3 & 50 & 635.1 & 699.4 & 676.6 & - \\
\hline & & 100 & 714.4 & 741.3 & 752.8 & - \\
\hline & & 200 & 754.8 & 793.2 & 798.8 & - \\
\hline & 5 & 50 & 542.2 & 644.7 & 621.5 & $(\eta, \lambda)_{\text {Eucl }}$ \\
\hline & & 100 & 546.3 & 665.8 & 649.7 & $(\eta, \lambda)_{\text {Tche }}$ \\
\hline & & 200 & 599.9 & 694.1 & 699.2 & $(\eta, \lambda)_{\text {Eucl }}$ \\
\hline & 7 & 50 & 523.5 & 610.2 & 612.8 & $(\eta, \lambda)_{\text {Eucl }}$ \\
\hline & & & & & \\
\hline & & & & & - \\
\hline & & & & & & - \\
\hline
\end{tabular}




\begin{tabular}{|c|c|c|c|c|c|c|}
\hline & & & & & & $(\eta, \lambda)_{\text {Tche }}$ \\
\hline & & 100 & 552.6 & 613.5 & 609.4 & - \\
\hline & & 200 & 586.7 & 636.4 & 620.5 & - \\
\hline & 9 & 50 & 475.7 & 592.4 & 576.2 & $\begin{array}{c}(\eta, \lambda)_{\text {Eucl }} \\
(\eta, \lambda)_{\text {Tche }}\end{array}$ \\
\hline & & 100 & 531.9 & 611.7 & 585.6 & $(\eta, \lambda)_{\text {Eucl }}$ \\
\hline & & 200 & 540.7 & 623.1 & 594.1 & $(\eta, \lambda)_{\text {Eucl }}$ \\
\hline 9 & 3 & 50 & 625.5 & 677.2 & 656.5 & - \\
\hline & & 100 & 643.6 & 692.4 & 687.2 & - \\
\hline & & 200 & 700.1 & 745.7 & 742.1 & - \\
\hline & 5 & 50 & 469.9 & 595.9 & 579.4 & $\begin{array}{c}(\eta, \lambda)_{\text {Eucl }} \\
(\eta, \lambda)_{\text {Tche }}\end{array}$ \\
\hline & & 100 & 551.1 & 611.4 & 608.6 & - \\
\hline & & 200 & 582.9 & 682.2 & 694.1 & $\begin{array}{c}(\eta, \lambda)_{\text {Eucl }} \\
(\eta, \lambda)_{\text {Tche }}\end{array}$ \\
\hline & 7 & 50 & 417.5 & 536.1 & 518.7 & $\begin{array}{c}(\eta, \lambda)_{\text {Eucl }} \\
(\eta, \lambda)_{\text {Tche }}\end{array}$ \\
\hline & & & & & & - \\
\hline & & 100 & 544.1 & 577.8 & 569.3 & - \\
\hline & 9 & 50 & 420.2 & 455.7 & 461.4 & - \\
\hline & & 100 & 444.5 & 477.5 & 472.8 & - \\
\hline & & 200 & 498.1 & 539.1 & 546.2 & - \\
\hline
\end{tabular}

* At least one of the null hypotheses was rejected.

Table 4. Average number of coincidences with $(\eta, \lambda) \operatorname{sim}$ (under additional constraints)

\begin{tabular}{|c|c|c|c|c|c|c|}
\hline$M$ & $N$ & card $(T)$ & $\begin{array}{c}\text { Ave. } \\
\text { coincidences } \\
(\eta, \lambda)_{\text {easy }}\end{array}$ & $\begin{array}{c}\text { Ave. } \\
\text { coincidences } \\
(\eta, \lambda)_{\text {Eucl }}\end{array}$ & $\begin{array}{c}\text { Ave. } \\
\text { coincidences } \\
(\eta, \lambda)_{\text {Tche }}\end{array}$ & $\begin{array}{c}\text { Better than } \\
(\eta, \lambda)_{\text {easy }}{ }^{*}\end{array}$ \\
\hline 3 & 3 & 50 & 899.3 & 897.4 & 896.1 & - \\
\hline & & 100 & 909.6 & 922.3 & 916.0 & - \\
\hline & & 200 & 935.2 & 942.4 & 940.6 & - \\
\hline & 5 & 50 & 870.0 & 868.5 & 862.0 & - \\
\hline & & 100 & 893.3 & 908.1 & 904.1 & - \\
\hline & & 200 & 923.1 & 920.4 & 918.2 & - \\
\hline & 7 & 50 & 842.4 & 832.9 & 834.2 & - \\
\hline & & 100 & 857.7 & 878.3 & 881.1 & - \\
\hline & & 200 & 907.1 & 905.9 & 915.8 & - \\
\hline & 9 & 50 & 782.9 & 813.0 & 820.9 & - \\
\hline & & 100 & 812.3 & 829.5 & 844.4 & - \\
\hline & & 200 & 880.6 & 899.4 & 908.9 & - \\
\hline 5 & 3 & 50 & 791.9 & 886.6 & 841.6 & $(\eta, \lambda)_{\text {Eucl }}$ \\
\hline & & 100 & 812.7 & 902.7 & 900.1 & $(\eta, \lambda)_{\text {Eucl }}$ \\
& & & & & & - \\
\hline & & 200 & 891.8 & 928.2 & 919.4 & - \\
\hline & 5 & 50 & 779.9 & 818.3 & 816.8 & - \\
\hline & & 100 & 825.3 & 869.0 & 872.0 & - \\
\hline & 200 & 848.5 & 899.7 & 891.3 & - \\
\hline & 7 & 50 & 706.7 & 745.0 & 750.2 & - \\
\hline
\end{tabular}




\begin{tabular}{|c|c|c|c|c|c|c|}
\hline & & 100 & 795.5 & 831.9 & 829.4 & - \\
\hline & & 200 & 833.7 & 862.4 & 867.0 & - \\
\hline & 9 & 50 & 711.3 & 743.0 & 751.6 & - \\
\hline & & 100 & 789.2 & 804.9 & 808.2 & - \\
\hline & & 200 & 813.0 & 833.7 & 839.1 & - \\
\hline \multirow[t]{12}{*}{7} & 3 & 50 & 787.2 & 873.7 & 842.4 & $(\eta, \lambda)_{\text {Eucl }}$ \\
\hline & & 100 & 815.6 & 897.8 & 869.9 & $(\eta, \lambda)_{\text {Eucl }}$ \\
\hline & & 200 & 850.7 & 922.2 & 898.9 & $(\eta, \lambda)_{\text {Eucl }}$ \\
\hline & 5 & 50 & 702.9 & 797.7 & 801.8 & $\begin{array}{l}(\eta, \lambda)_{\text {Eucl }} \\
(\eta, \lambda)_{\text {Tche }}\end{array}$ \\
\hline & & 100 & 757.2 & 859.6 & 844.8 & $\begin{array}{l}(\eta, \lambda)_{\text {Eucl }} \\
(\eta, \lambda)_{\text {Tche }}\end{array}$ \\
\hline & & 200 & 811.7 & 894.4 & 861.9 & $(\eta, \lambda)_{\text {Eucl }}$ \\
\hline & 7 & 50 & 676.1 & 720.3 & 718.3 & - \\
\hline & & 100 & 714.3 & 794.8 & 796.4 & $\begin{array}{l}(\eta, \lambda)_{\text {Eucl }} \\
(\eta, \lambda)_{\text {Tche }}\end{array}$ \\
\hline & & 200 & 792.9 & 838.0 & 834.5 & - \\
\hline & 9 & 50 & 581.4 & 669.1 & 664.4 & $\begin{array}{l}(\eta, \lambda)_{\text {Eucl }} \\
(\eta, \lambda)_{\text {Tche }}\end{array}$ \\
\hline & & 100 & 678.5 & 725.5 & 730.1 & - \\
\hline & & 200 & 729.1 & 781.3 & 802.2 & $(\eta, \lambda)_{\text {Tche }}$ \\
\hline \multirow[t]{12}{*}{9} & 3 & 50 & 761.3 & 843.2 & 841.4 & $\begin{array}{l}(\eta, \lambda)_{\text {Eucl }} \\
(\eta, \lambda)_{\text {Tche }}\end{array}$ \\
\hline & & 100 & 793.4 & 870.1 & 845.3 & $(\eta, \lambda)_{\text {Eucl }}$ \\
\hline & & 200 & 832.3 & 914.6 & 877.2 & $(\eta, \lambda)_{\text {Eucl }}$ \\
\hline & 5 & 50 & 700.1 & 784.3 & 792.6 & $\begin{array}{l}(\eta, \lambda)_{\text {Eucl }} \\
(\eta, \lambda)_{\text {Tche }}\end{array}$ \\
\hline & & 100 & 757.1 & 846.6 & 813.1 & $(\eta, \lambda)_{\text {Eucl }}$ \\
\hline & & 200 & 810.6 & 889.2 & 851.9 & $(\eta, \lambda)_{\text {Eucl }}$ \\
\hline & 7 & 50 & 596.2 & 671.3 & 678.1 & $\begin{array}{l}(\eta, \lambda)_{\text {Eucl }} \\
(\eta, \lambda)_{\text {Tche }}\end{array}$ \\
\hline & & 100 & 685.8 & 770.7 & 774.4 & $\begin{array}{l}(\eta, \lambda)_{\text {Eucl }} \\
(\eta, \lambda)_{\text {Tche }}\end{array}$ \\
\hline & & 200 & 777.2 & 809.5 & 810.9 & - \\
\hline & 9 & 50 & 560.6 & 638.8 & 601.5 & $(\eta, \lambda)_{\text {Eucl }}$ \\
\hline & & 100 & 667.8 & 707.1 & 703.7 & - \\
\hline & & 200 & 711.4 & 769.6 & 762.1 & - \\
\hline
\end{tabular}

* At least one of the null hypotheses was rejected. 